\title{
A NOTE ON INVERSE LIMITS OF CONTINUOUS IMAGES OF ARCS
}

\author{
IVAN LONČAR
}

\begin{abstract}
The main purpose of this paper is to prove some theorems concerning inverse systems and limits of continuous images of arcs. In particular, we shall prove that if $\mathbf{X}=\left\{X_{a}, p_{a b}, A\right\}$ is an inverse system of continuous images of arcs with monotone bonding mappings such that $\operatorname{cf}(\operatorname{card}(A)) \neq \omega_{1}$, then $X=\lim \mathbf{X}$ is a continuous image of an arc if and only if each proper subsystem $\left\{X_{a}, p_{a b}, B\right\}$ of $\mathbf{X}$ with $\operatorname{cf}(\operatorname{card}(B))=\omega_{1}$ has the limit which is a continuous image of an arc (Theorem 18).
\end{abstract}

\section{Inverse limits of hereditarily locally connected continua}

An arc (or ordered continuum) is a Hausdorff continuum with exactly two non-separating points. Each separable arc is homeomorphic to the closed interval $I=[0,1]$.

A space $X$ is said to be an IOK (IOC) if there exists an ordered compact (connected) space $K$ and a continuous surjection $f: K \rightarrow X$. Frequently, we will say that a space $X$ is a continuous image of an arc if $X$ is an IOC.

The cardinality of a set $A$ will be denoted by $\operatorname{card}(A)$. We assume that $\operatorname{card}(A)$ is the initial ordinal number. The cofinality of a cardinal number $m$ will be denoted by $\mathrm{cf}(m)$.

Keywords. Inverse system and limit, continuous image of an arc.

1991 Mathematics subject classifications: 54B35, 54C05, 54F50. 
A continuum $X$ is said to be hereditarily locally connected if each subcontinuum of $X$ is locally connected. A continuum $X$ is said to be finitely Suslinian [17] if there do not exist open sets $U$ and $V$, and an infinite collection $\mathcal{K}$ of pairwise disjoint subcontinua of $X$ such that $C l(U) \cap C l(V)=\emptyset$ and $K \cap V \neq \emptyset$ and $K \cap U \neq \emptyset$ for each $K$ in $\mathcal{K}$. Each finitely Suslinian continuum is hereditarily locally connected. A continuum $X$ is rim-finite (rim-countable) if it has a basis $\mathcal{B}$ such that $\operatorname{card}(\operatorname{Bd}(U))<\aleph_{0}\left(\operatorname{card}(\operatorname{Bd}(U)) \leq \aleph_{0}\right)$ for each $U \in \mathcal{B}$. Each rimfinite continuum is finitely Suslinian. Each hereditarily locally connected continuum is a continuous image of an arc [11, Theorem 3.4].

In the paper [8, Problem 9.10] the authors asked when the inverse limit of an inverse system $\mathbf{X}=\left\{X_{a}, p_{a b}, A\right\}$ of hereditarily locally connected continua with monotone surjective bonding mappings $p_{a b}$ is a continuous image of an arc.

If $\mathbf{X}=\left\{X_{a}, p_{a b}, A\right\}$ is an inverse system of hereditarily locally connected continua, then $X=\lim \mathbf{X}$ need not be a hereditarily locally continuum since each locally connected metric continuum of dimension 1 (= curve) is the limit of an inverse sequence of rim-finite continua with surjective monotone bonding mappings [13, Theorem 2.2].

In the present section we shall define a class of hereditarily locally connected continua such that each inverse limit of such spaces and monotone bonding mappings has a hereditarily locally connected limit.

In Appendix we review some definitions and known results needed in this section.

We say that an inverse system $\mathbf{Y}=\left\{Y_{a}, q_{a b}, B\right\}$ is a subsystem of $\mathbf{X}=\left\{X_{a}, p_{a b}, A\right\}$ if $B \subset A, Y_{a}=X_{a}$ and each $q_{a b}$ is $p_{a b}$.

We start with the following theorem.

Theorem 1. Let $X$ be the limit of an inverse system $\mathbf{X}=\left\{X_{a}, p_{a b}, A\right\}$ of hereditarily locally connected continua $X_{a}$ such that the bonding mappings $p_{a b}: X_{b} \rightarrow X_{a}$ are monotone surjections. Then $X$ is a hereditarily locally continuum if and only if each countable inverse subsystem of $\mathbf{X}$ has a hereditarily locally connected limit.

Proof: By Theorem $29 X$ is homeomorphic to the limit of $\mathbf{X}_{\sigma}=$ $\left\{X_{\Delta}, p_{\Delta \Gamma}, A_{\sigma}\right\}$, where $A_{\sigma}$ is the family of all nonempty countable directed subsets of $A$. If $X$ is hereditarily locally connected then each $X_{\Delta}$ is hereditarily locally connected as a monotone image of a hereditarily locally connected continuum $X$ (see Lemma 28). Conversely, if each $X_{\Delta}$ is hereditarily locally connected, then $X$ is hereditarily locally connected (Theorem 27). 
The following theorem is a generalization of the well-known result of G. T. Whyburn [20, p. 81] which asserts that a metric continuum $X$ is hereditarily locally connected if and only if each cyclic element (see Appendix) $Z \subseteq X$ is hereditarily locally connected.

Theorem 2. A locally connected continuum $X$ is hereditarily locally connected if and only if each cyclic element of $X$ is hereditarily locally connected.

Proof: By Theorems 31 and 29 there exists a $\sigma$-directed inverse system $\mathbf{X}=\left\{X_{a}, p_{a b}, A\right\}$ of metric locally connected spaces such that $p_{a b}$ are monotone and $X$ is homeomorphic to $\lim \mathbf{X}$. Let us prove that each $X_{a}$ is hereditarily locally connected. It suffices to prove that each cyclic element $Z_{a}$ of $X_{a}$ is hereditarily locally connected. By Lemma 34 there exists a cyclic element $Z$ of $\lim \mathbf{X}$ such $p_{a}(Z) \supseteq Z_{a}$. Since $\lim \mathbf{X}$ is homeomorphic to $X, Z$ is hereditarily locally connected. This means that $p_{a}(Z)$ is hereditarily locally connected. It follows that $Z_{a}$ is hereditarily locally connected since $Z_{a} \subseteq p_{a}(Z)$. We infer that each $X_{a}$ is hereditarily locally connected since $X_{a}$ is a metric continuum. From Theorem 27 it follows that $X$ is hereditarily locally connected.

A surjective mapping $f: X \rightarrow Y$ is said to be hereditarily monotone $[\mathbf{5}, \mathrm{pp} .16-17]$ if for each subcontinuum $K$ of $X$ the restriction $f \mid K$ : $K \rightarrow f(K)$ is monotone.

If $f: X \rightarrow Y$ and $g: Y \rightarrow Z$ are hereditarily monotone mappings, then $g f: X \rightarrow Z$ is hereditarily monotone $[\mathbf{5}$, p. 29, (5.3)].

Lemma 3. If $Z$ is a cyclic element of $X$, then the canonical retraction $\rho: X \rightarrow Z$ is hereditarily monotone.

Proof: Let $\rho: X \rightarrow Z$ (see Appendix, p. 13) and let $K$ be any subcontinuum of $X$. Let us prove that the restriction $\rho_{K}: K \rightarrow \rho_{K}(K) \subseteq Z$ is monotone. Consider the following cases.

a) $K \subseteq Z$. Now $\rho_{K}$ is the identity and is monotone.

b) $K \subseteq X \backslash Z$. It is clear that $K$ is a subset of some component $J$ of $X \backslash Z$. Let $\left\{z_{J}\right\}=\operatorname{Bd}(J)$. Now, $\rho_{K}(K)=\left\{z_{J}\right\}$. This means that $\rho_{K}^{-1}\left(z_{J}\right)=K$. Hence $\rho_{K}$ is monotone.

c) $K \bigcap Z \neq \emptyset$ and $K \bigcap(X \backslash Z) \neq \emptyset$. In this case

$K=(K \bigcap Z) \bigcup\{K \bigcap J: J$ is a component of $X \backslash Z, K \bigcap J \neq \emptyset\}$ 
We infer that

$\rho_{K}(K)=(K \bigcap Z) \bigcup\left\{\left\{z_{J}\right\}: J\right.$ is a component of $\left.X \backslash Z,\left\{z_{J}\right\}=\operatorname{Bd}(J)\right\}$.

If $x \in \rho_{K}(K)$ such that $x \neq z_{J}$ for all components $J$ of $X \backslash Z$, then $\rho_{K}^{-1}(x)=\{x\}$. Hence $\rho_{K}^{-1}(x)$ is connected. If $x=z_{J}$ for some component $J$, then

$$
\rho_{K}^{-1}(x)=\left\{z_{J}\right\} \bigcup\left\{K \bigcap J_{i}:\left\{z_{J}\right\}=\operatorname{Bd}\left(J_{i}\right), i \in I\right\}
$$

It suffices to prove that each set $C l\left(J_{i}\right) \bigcap K$ is connected. Suppose that $C l\left(J_{i}\right) \bigcap K$ is not connected. There exists a component $L$ of $C l\left(J_{i}\right) \bigcap K$ such that $z_{J} \notin L$. By virtue of the normality of $X$ it follows that there exists a pair $U, V$ of disjoint open sets such that $L \subseteq U$ and $z_{J} \in V$. We may assume that $C l U \subseteq J$ since $J$ is open. For each point $x$ of $K \backslash(U \bigcup V)$ there exists an open set $U_{x}$ such that $x \in U_{x}$ and $U_{x} \bigcap U=\emptyset$. Let $W$ be the union of $V$ and all the sets $U_{x}, x \in K \backslash(U \cup V)$. It is clear that $U \cap W=\emptyset$ and $K \subseteq U \bigcup W$. This is impossible since $K$ is connected. Hence, $C l\left(J_{i}\right) \bigcap K$ is connected and $\rho_{K}$ is monotone.

Theorem 4. Let $\mathbf{X}=\left\{X_{a}, p_{a b}, A\right\}$ be an inverse system of continua and hereditarily monotone bonding mappings. Then the projections $p_{a}$, $a \in A$, are hereditarily monotone. Moreover, if each $X_{a}$ is hereditarily locally connected, then $X=\lim \mathbf{X}$ is hereditarily locally connected.

Proof: Let $K$ be a subcontinuum of $X$. For each $a \in A, K_{a}=p_{a}(K)$ is a subcontinuum of $X_{a}$. We have the inverse system $K=\left\{K_{a}, p_{a b} \mid\right.$ $\left.K_{b}, A\right\}$. From the definition of hereditarily monotone mapping it follows that each mapping $p_{a b} \mid K_{b}$ is a monotone mapping. This means that the projections $p_{a} \mid K$ are monotone [2, pp. 462-463]. From [2, Theorem 6.1.28] it follows that $K$ is locally connected. Thus, $X$ is hereditarily locally connected.

A surjective mapping $f: X \rightarrow Y$ is said to be cyclically hereditarily monotone if for each cyclic element $Z$ of $X$ the restriction $f \mid Z$ is hereditarily monotone.

Theorem 5. Let $\mathbf{X}=\left\{X_{a}, p_{a b}, A\right\}$ be an inverse system of hereditarily locally connected continua $X_{a}$ and cyclically hereditarily monotone bonding mappings $p_{a b}$. Then $X=\lim \mathbf{X}$ is hereditarily locally connected. 
Proof: By Theorem 2 it suffices to prove that each cyclic element $Z$ of $X$ is hereditarily locally connected. There exists an inverse system (Theorem 35) $\mathbf{Z}=\left\{Z_{a}, g_{a b}, A\right\}$ such that $Z_{a}$ is a cyclic element of $X_{a}$, $g_{a b}=\rho_{a} \circ\left(f_{a b} \mid Z_{b}\right)$ for all $a \leq b \in A$, and $Z$ is homeomorphic to $\lim \mathbf{Z}$. This means that $g_{a b}$ is hereditarily monotone since $\rho_{a}$ is hereditarily monotone (Lemma 3). From Theorem 4 it follows that $Z$ is hereditarily locally connected. Thus, $X$ is hereditarily locally connected.

A space $X$ is in class $\mathcal{H}_{m}$ if $X$ is a hereditarily locally connected continuum and $X$ contains no non-degenerate metric subcontinuum. Each space in class $\mathcal{H}_{m}$ is rim-finite [19, Theorem 1$]$.

Theorem 6. Let $\mathbf{X}=\left\{X_{n}, p_{m n}, \mathbb{N}\right\}$ be an inverse sequence with monotone surjective bonding mappings. If each $X_{n}$ is in class $\mathcal{H}_{m}$, then $X=\lim \mathbf{X}$ is in class $\mathcal{H}_{m}$. Moreover, $X$ is rim-finite.

Proof: Each $X_{n}$ is a continuous image of an arc [11, Corollary 3.5] since each $X_{n}$ is hereditarily locally connected. Thus, $X$ is a continuous image of an arc (Theorem 14). Let us prove that $X$ contains no nondegenerate metric subcontinuum. Suppose that $Y$ is a non-degenerate metric subcontinuum of $X$. Then there exists a $n \in N$ such that $p_{m}(Y)$ is a non-degenerate metric subcontinuum of $X_{m}$ for each $m \geq n$. This is impossible since $X_{m}$ is in class $\mathcal{H}_{m}$. We infer that $X$ contains no non-degenerate metric subcontinua. By virtue of Theorem 21 it follows that $X$ is hereditarily locally connected. Moreover, from Theorem 1 of [19] it follows that $X$ is rim-finite.

Theorem 7. Let $\mathbf{X}=\left\{X_{a}, p_{a b}, A\right\}$ be an inverse system with monotone surjective bonding mappings. If each $X_{a}$ is in class $\mathcal{H}_{m}$, then $X=\lim \mathbf{X}$ is in class $\mathcal{H}_{m}$. Moreover, $X$ is rim-finite.

Proof: Apply Theorem 1 and Theorem 23.

A hereditarily locally connected continuum $X$ is in class $\mathcal{H}_{z m}$ if each cyclic element $Z$ of $X$ is in class $\mathcal{H}_{m}$.

Theorem 8. Let $\mathbf{X}=\left\{X_{a}, p_{a b}, A\right\}$ be an inverse system with monotone surjective bonding mappings. If each $X_{a}$ is in class $\mathcal{H}_{z m}$, then $X=\lim X$ is in class $\mathcal{H}_{z m}$.

Proof: Let $Z$ be a cyclic element of $X$. By Theorem 35 there exists an inverse system $\left(Z_{\gamma}, g_{\gamma \gamma^{\prime}}, \Gamma\right)$ such that $Z$ is homeomorphic to $\lim \operatorname{inv}\left(Z_{\gamma}, g_{\gamma \gamma^{\prime}}, \Gamma\right)$, where $Z_{\gamma}$ is a cyclic element of $X_{\gamma}$ and $g_{\gamma \gamma^{\prime}}$ is monotone. By Theorem $7 Z$ is in class $\mathcal{H}_{m}$. From Theorem 2 it follows that $X$ is hereditarily locally connected. Hence, $X$ is in class $\mathcal{H}_{z m}$. 


\section{Special classes of continuous images of arcs}

Theorem $9[\mathbf{9}]$. Let $X$ be a locally connected continuum such that for each pair of distinct points $a, b$ in $X$, there exists a continuous onto map $f: X \rightarrow[c, d]$ such that $f(a)=c$ and $f(b)=d$ and $[c, d]$ is a nonmetrizable arc. If $X$ is rim-metrizable or rim-scattered or monotonically normal, then $X$ is a continuous image of an arc.

A locally connected continuum is said to be a NTT-space if for each pair of distinct points $a, b$ in $X$, there exists a continuous onto map $f$ : $X \rightarrow[c, d]$ such that $f(a)=c$ and $f(b)=d$ and $[c, d]$ is a non-metrizable arc.

Theorem 10. Let $\mathbf{X}=\left\{X_{a}, p_{a b}, A\right\}$ be an inverse system of NTTspaces with monotone surjective bonding mappings. Then $X=\lim \mathbf{X}$ is a NTT-space.

Proof: It is known that $X$ is locally connected continuum. Let $x, y$ be a pair of distinct points of $X$. There exists an $a \in A$ such that $p_{b}(x) \neq p_{b}(y)$ for each $b \geq a$. Since $X_{b}$ is a NTT-space there exists a non-metrizable arc $[c, d]$ and a surjective mapping $f: X_{b} \rightarrow[c, d]$ such that $f(x)=c$ and $f(y)=d$. Considering the mapping $f p_{b}: X \rightarrow[c, d]$ we infer that $X$ is a NTT-space.

Theorem 11. Let $\mathbf{X}=\left\{X_{a}, p_{a b}, A\right\}$ be an inverse system of NTTspaces and monotone surjective bonding mappings. If $X=\lim \mathbf{X}$ is rim-metrizable or rim-scattered or monotonically normal, then $X$ is a continuous image of an arc.

Proof: By Theorem $10 X$ is a NTT-space. Apply Theorem 9.

Theorem 12. Let $\mathbf{X}=\left\{X_{a}, p_{a b}, A\right\}$ be a $\sigma$-directed inverse system of spaces $X_{a}$ such that for each pair $x_{a}$, $y_{a}$ of points of $X_{a}$ the subspace $X_{a} \backslash\left\{x_{a}, y_{a}\right\}$ is connected, $a \in A$. If each $X_{a}$ is a continuous image of an arc and each $p_{a b}$ is a monotone surjection, then $X=\lim \mathbf{X}$ is a continuous image of an arc if and only if there exists an $a \in A$ such that $p_{b}: X \rightarrow X_{b}$ is a homeomorphism for each $b \geq a$ (if and only if $X$ is metrizable).

Proof: By Theorem 2 of [18] each $X_{a}$ is metrizable. We shall prove that for each pair $x, y$ of points of $X$ the subspace $Y=X \backslash\{x, y\}$ is connected. Suppose that $Y$ is not connected. Then there exists a pair $U, V$ of disjoint open subsets of $X$ such that $Y=U \cup V$. Moreover, 
there exists an $a \in A$ such that $p_{b}(x) \neq p_{b}(y), b \geq a$. The sets $U_{a}=$ $\left\{x_{a}: x_{a} \in X_{a}, p_{a}^{-1}\left(x_{a}\right) \subset U\right\}$ and $V_{a}=\left\{x_{a}: x_{a} \in \bar{X}_{a}, p_{a}^{-1}\left(x_{a}\right) \subset V\right\}$ are disjoint and open. Now we have $X \backslash\left\{p_{a}(x), p_{a}(y)\right\}=U_{a} \cup V_{a}$. This is impossible since $X \backslash\left\{p_{a}(x), p_{a}(y)\right\}$ is connected. Hence, $Y=X \backslash\{x, y\}$ is connected. It follows that if $X$ is a continuous image of an arc, then it is metrizable $([\mathbf{1 8}$, Theorem 2]). From Theorem 26 it follows that there exists a $b \in A$ such that $p_{c}: X \rightarrow X_{c}$ is a homeomorphism for every $c \geq b$. Conversely, if such $b \in A$ exists, then $X$ is a continuous image of an arc.

Theorem 13. Let $\mathbf{X}=\left\{X_{a}, p_{a b}, A\right\}$ be an inverse system of spaces $X_{a}$ such that for each pair $x_{a}, y_{a}$ of points of $X_{a}$ the subspace $X_{a} \backslash\left\{x_{a}, y_{a}\right\}$ is connected, $a \in A$. If each $X_{a}$ is a continuous image of an arc and each $p_{a b}$ is a monotone surjection, then $X=\lim \mathbf{X}$ is a continuous image of an arc if and only if there exists a countable subsystem $\mathbf{Y}$ of $\mathbf{X}$ such that $\lim \mathbf{Y}$ is homeomorphic to $X$.

Proof: Consider the inverse system $\mathbf{X}_{\sigma}=\left\{X_{\Delta}, p_{\Delta \Gamma}, A_{\sigma}\right\}$ from Theorem 29 and apply Theorem 12.

\section{Inverse systems and subsystems}

Theorem $14\left[\mathbf{8}\right.$, Theorem 5.1]. Let $\mathbf{X}=\left\{X_{n}, p_{m n}, \mathbb{N}\right\}$ be an inverse sequence with monotone surjective bonding mappings. If each $X_{n}$ is the continuous image of an arc, then $X=\lim \mathbf{X}$ is the continuous image of an arc.

Theorem 15 [4, Theorem 2.17]. Let $\mathbf{X}=\left\{X_{a}, p_{a b}, A\right\}$ be a wellordered inverse system such that $\operatorname{cf}(A) \neq \omega_{1}$. If the mappings $p_{a b}$ are monotone surjections and if the spaces $X_{a}$ are the continuous images of arcs, then $X=\lim \mathbf{X}$ is the continuous image of an arc.

Remark 16. Theorem 15 is not true if $\operatorname{cf}(A)=\omega_{1}$. This is shown by the following example of Nikiel [10]. Let $L$ denote the long interval $[\mathbf{2}$, p. 297]. For each ordinal number $\alpha, 0<\alpha<\omega_{1}$, let $f_{\alpha}:[0,1] \times L \rightarrow$ $[0,1] \times[0, \alpha]_{L}$ be defined by

$$
f(s, t)= \begin{cases}(s, t) & \text { if } t \leq_{L} \alpha \\ (s, \alpha) & \text { if } \alpha \leq_{L} t .\end{cases}
$$

Each $X_{\alpha}=[0,1] \times[0, \alpha]_{L}$ is homeomorphic to $[0,1] \times[0,1]$ and it is a continuous image of an arc. Moreover, $w\left(X_{a}\right)=\aleph_{0}$. Let $f_{\alpha \beta}=f_{\alpha}$ $[0,1] \times[0, \beta]_{L}, \beta<\alpha$. We obtain an inverse system $\left\{X_{\alpha}, f_{\alpha \beta}, \alpha<\omega_{1}\right\}$ whose limit is $[0,1] \times L$ which is not a continuous image of an arc. 
Theorem 17. Let $\mathbf{X}=\left\{X_{a}, p_{a b}, A\right\}$ be an inverse system of compact spaces such that $\operatorname{card}(A)>\aleph_{0}$. There exists a transfinite sequence $\left\{A_{\alpha}\right.$ : $\alpha<\operatorname{card}(A)\}$ of directed subsets $A_{\alpha}$ of $A$ such that:

1. $\operatorname{card}\left(A_{\alpha}\right)<\operatorname{card}(A), \alpha<\operatorname{card}(A)$,

2. $\alpha<\beta<\operatorname{card}(A)$ implies $A_{\alpha} \subseteq A_{\beta}$,

3. $A=\bigcup\left\{A_{\alpha}: \alpha<\operatorname{card}(A)\right\}$,

4. each collection $\left\{X_{a}, p_{a b}, A_{\alpha}\right\}$ is an inverse system with limit $X_{\alpha}$,

5. if $\alpha<\beta<\operatorname{card}(A)$ then there exists a mapping $q_{\alpha \beta}: X_{\beta} \rightarrow X_{\alpha}$,

6. $\lim \mathbf{X}$ is homeomorphic to $\lim \left\{X_{\alpha}, q_{\alpha \beta}, \alpha<\beta<\operatorname{card}(A)\right\}$,

7. if the mappings $p_{a b}$ are monotone, then the mappings $q_{\alpha \beta}$ are monotone.

Proof: The proof consists of several steps. Step 1 is from $[\mathbf{7}$, pp. 238239, Hilfssatz]. For the sake of the completeness we give the proof of Step 1.

Step 1: Let $\nu$ be any finite subset of $A$. There exists a $\delta(\nu) \in A$ such that $\delta \leq \delta(\nu)$ for each $\delta \in \nu$. For each $B \subseteq A$ there exists a set $F_{1}(B)=B \bigcup\{\delta(\nu): \nu \subset B$ and $\nu$ is finite $\}$. Put

$$
F_{n+1}=F_{1}\left(F_{n}(B)\right)
$$

and

$$
F_{\infty}(B)=\bigcup\left\{F_{n}(B): n \in \mathbb{N}\right\}
$$

It is clear that

$$
F_{1}(B) \subseteq F_{2}(B) \subseteq \ldots \subseteq F_{n}(B) \subseteq \cdots
$$

The set $F_{\infty}(B)$ is directed since each finite subset $\nu$ of $F_{\infty}(B)$ is contained in some $F_{n}(B)$ and, consequently, $\delta(\nu)$ is contained in $F_{\infty}(B)$. If $B$ is finite, then $\operatorname{card}\left(F_{\infty}(B)\right)=\aleph_{0}$. If $\operatorname{card}(B) \geq \aleph_{0}$, then we have $\operatorname{card}(\{\delta(\nu): \nu \in B\}) \leq \operatorname{card}(B) \aleph_{0}$. We infer that $\operatorname{card}\left(F_{1}(B)\right) \leq$ $\operatorname{card}(B) \aleph_{0}$. Similarly, $\operatorname{card}\left(F_{n}(B)\right) \leq \operatorname{card}(B) \aleph_{0}$. This means that $\operatorname{card}\left(F_{\infty}(B)\right) \leq \operatorname{card}(B) \aleph_{0}$. Thus

$$
\operatorname{card}\left(F_{\infty}(B)\right) \leq \operatorname{card}(B) \aleph_{0} .
$$

Suppose that $\operatorname{card}(A)>\aleph_{0}$. Put $\Omega=\operatorname{card}(A)$. Hence, $A=\left\{a_{\alpha}: \alpha<\right.$ $\Omega\}$. Put $B_{\alpha}=\left\{a_{\mu}: \mu<\alpha<\Omega\right\}$. We have a transfinite sequence $\left\{B_{\alpha}: \alpha<\Omega\right\}$ such that
a) $\operatorname{card}\left(B_{\alpha}\right)<\operatorname{card}(A)$,
b) $\alpha<\beta<\Omega$ implies $B_{\alpha} \subseteq B_{\beta}$,
c) $A=\bigcup\left\{B_{\alpha}: \alpha<\Omega\right\}$.

Put $A_{\alpha}=F_{\infty}\left(B_{\alpha}\right)$. 
Step 2: Assertions 1-3 follow from Step 1.

Step 3: Assertion 4 follows from the fact that each $A_{\alpha}$ is directed subset of $A$.

Step 4: Let us prove 5. From assertion 2 it follows that there exists a continuous mapping $q_{\alpha \beta}: X_{\beta} \rightarrow X_{\alpha}$ since each point $x \in X_{\beta}$ induces a collection $\left\{x_{a}: a \in A_{\alpha}\right\}$ which satisfies $p_{a b}\left(x_{b}\right)=x_{a}$, i.e., $\left\{x_{a}: a \in A_{\alpha}\right\}$ is a point of $X_{\alpha}$.

Step 5: It is obvious that there exists a mapping $H: \lim \mathbf{X} \rightarrow$ $\lim \left\{X_{\alpha}, q_{\alpha \beta}, \alpha<\beta<\operatorname{card}(A)\right\}$ since each $x=\left(x_{a}: a \in A\right) \in \lim \mathbf{X}$ induces a collection $\left\{x_{a}: a \in A_{\alpha}\right\}$ on each $A_{\alpha}$. Thus we have the mappings $H_{\alpha}: \lim \mathbf{X} \rightarrow X_{\alpha}$, for each $\alpha<\operatorname{card}(A)$. The mappings $H_{\alpha}$ induce a continuous mapping $H: \lim \mathbf{X} \rightarrow \lim \left\{X_{\alpha}, q_{\alpha \beta}, \alpha<\beta<\operatorname{card}(A)\right\}$. It remains to prove that $H$ is $1-1$ and onto. Let us prove that $H$ is 1-1. Let $x, y \in \lim \mathbf{X}$ and $x \neq y$. There exists an $a \in A$ such that $x_{a} \neq y_{a}$. From Step 1 it follows that there is an $A_{\alpha}$ such that $a \in A_{\alpha}$. Now, $x_{a} \neq y_{a}$ implies $H_{\alpha}(x) \neq H_{\alpha}(y)$ (see Step 5). This means that $H(x) \neq H(y)$. Hence $H$ is 1-1. In order to complete the proof it suffices to prove that $H$ is onto. Let $y=\left(y_{\alpha}: \alpha<\operatorname{card}(A)\right)$ be any point of $\lim \left\{X_{\alpha}, q_{\alpha \beta}, \alpha<\beta<\operatorname{card}(A)\right\}$. Then $y_{\alpha} \in X_{\alpha}$. Thus, $y_{\alpha}$ is a thread in $X_{\alpha}$, i.e., $y=\left(x_{a}, a \in A_{\alpha}\right)$. We infer that for each $a \in A$ there exists a point $x_{a} \in X_{a}$. It is readily to seen that $p_{a b}\left(x_{b}\right)=x_{a}$. Thus, $\left(x_{a}: a \in A\right)$ is a thread in $\lim \mathbf{X}$ such that $H(x)=y$.

Step 6: Let us prove 7. If the mappings $p_{a b}$ are monotone, then from Lemma 28 it follows that the mappings $q_{\alpha \beta}$ are monotone.

Now we shall prove the main theorem of this section which is a generalization of Theorem 15 .

Theorem 18. Let $\mathbf{X}=\left\{X_{a}, p_{a b}, A\right\}$ be an inverse system of continuous images of arcs with monotone bonding mappings. If $\operatorname{cf}(\operatorname{card}(A)) \neq$ $\omega_{1}$, then $X=\lim \mathbf{X}$ is a continuous image of an arc if and only if each proper subsystem $\left\{X_{a}, p_{a b}, B\right\}$ of $\mathbf{X}$ with $\operatorname{cf}(\operatorname{card}(B))=\omega_{1}$ has the limit which is a continuous image of an arc.

Proof: The "only if part". If $X$ is a continuous image of an arc, then for each subsystem $\left\{X_{a}, p_{a b}, B\right\}$ there exists a natural projections $f_{a}: X \rightarrow \lim \left\{X_{a}, p_{a b}, B\right\}$. Hence, $\lim \left\{X_{a}, p_{a b}, B\right\}$ is a continuous image of an arc. 
The "if" part. By Theorem 17 there exists a well-ordered inverse system $\left\{X_{\alpha}, q_{\alpha \beta}, \alpha<\beta<\operatorname{card}(A)\right\}$ such that $X$ is homeomorphic to $\lim \left\{X_{\alpha}, q_{\alpha \beta}, \alpha<\beta<\operatorname{card}(A)\right\}$. If $\operatorname{cf}(\operatorname{card}(A)) \leq \omega_{0}$, then we have an inverse subsequence of $\left\{X_{\alpha}, q_{\alpha \beta}, \alpha<\beta<\operatorname{card}(A)\right\}$ which is a cofinal subsystem of $\left\{X_{\alpha}, q_{\alpha \beta}, \alpha<\beta<\operatorname{card}(A)\right\}$. By Theorem $14 X$ is a continuous image of an arc. Now, suppose that $\operatorname{cf}(\operatorname{card}(A))>\omega_{1}$. By Theorem 17 it suffices to prove that each subsystem of $\left\{X_{a}, p_{a b}, B\right\}$ of $\mathbf{X}=\left\{X_{a}, p_{a b}, A\right\}$ has the limit which is a continuous image of an arc. We shall use the transfinite induction on $\operatorname{card}(B)$. If $\operatorname{card}(B) \leq \omega_{0}$, then we use Theorem 14. If $\operatorname{card}(B)=\omega_{1}$, then $\lim \left\{X_{a}, p_{a b}, B\right\}$ is a continuous image of an arc by assumption of Theorem. Let now $\left\{X_{a}, p_{a b}, B\right\}$ be a subsystem of $\left\{X_{a}, p_{a b}, A\right\}$ such that $\operatorname{card}(B)>\omega_{1}$. Suppose that Theorem is true for each subsystem of the cardinality $<\operatorname{card}(B)$. By Theorem 17 there exists a well-ordered inverse system $\left\{X_{\alpha}, q_{\alpha \beta}, \alpha<\beta<\operatorname{card}(B)\right\}$ such that $\lim \left\{X_{a}, p_{a b}, B\right\}$ is homeomorphic to $\lim \left\{X_{\alpha}, q_{\alpha \beta}, \alpha<\beta<\operatorname{card}(B)\right\}$. Since each $X_{\alpha}$ is the limit of a subsystem of the cardinality $<\operatorname{card}(B)$, we have the inverse system $\left\{X_{\alpha}, q_{\alpha \beta}, \alpha<\beta<\operatorname{card}(B)\right\}$ which satisfies the conditions of Theorem 15. Thus, $\lim \left\{X_{a}, p_{a b}, B\right\}$ is a continuous image of an arc. By the transfinite induction the proof is complete.

Corollary 19. Let $X$ be a locally connected continuum. The following conditions are equivalent:

a) $X$ is a continuous image of an arc.

b) If $f: X \rightarrow Y$ is a continuous mapping and $\operatorname{cf}(w(Y))=\omega_{1}$, then $Y$ is a continuous image of an arc.

Proof: a) $\Rightarrow$ b). Obvious.

b) $\Rightarrow$ a). By Theorem 31 there exists an inverse system $\mathbf{X}=\left\{X_{a}, p_{a b}, A\right\}$ such that $X_{a}$ are metric locally connected continua, $p_{a b}$ are monotone mappings and $X$ is homeomorphic to $\lim \mathbf{X}$. If $\mathbf{Y}=\left\{X_{a}, p_{a b}, B\right\}$ is any subsystem of $\left\{X_{a}, p_{a b}, A\right\}$ with $\operatorname{cf}(\operatorname{card}(B))=\omega_{1}$, then there exists a natural projection $P: X \rightarrow \lim \mathbf{Y}$. By b) it follows that $\lim \mathbf{Y}$ is a continuous image of an arc since $w(\lim \mathbf{Y})=\aleph_{1}$. Applying Theorem 18 we complete the proof.

Corollary 20. Let $X$ be a locally connected continuum such that $w(X)>\aleph_{\omega_{1}}$. The following conditions are equivalent:

a) $X$ is a continuous image of an arc.

b) If $f: X \rightarrow Y$ is a continuous mapping and $w(Y)=\aleph_{1}$, then $Y$ is a continuous image of an arc. 


\section{Appendix}

Theorem 21 [15, Theorem 4]. If $X$ is a connected IOK and $X$ contains no nondegenerate metric subcontinuum, then $X$ is hereditarily locally connected.

Theorem 22 [17, Theorem]. If $X$ is a continuum containing no nondegenerate metric subcontinuum, then $X$ is finitely Suslinian if and only if $X$ is a continuous image of an arc.

We say that $\mathbf{X}=\left\{X_{a}, p_{a b}, A\right\}$ is $\sigma$-directed if for each sequence $a_{1}, a_{2}, \ldots, a_{k}, \ldots$ of the members of $A$ there is an $a \in A$ such that $a \geq a_{k}$ for each $k \in \mathbb{N}$.

Theorem 23 [8, Theorem 9.8]. If $X$ is the limit of a $\sigma$-directed inverse system of finitely Suslinian continua, then $X$ is finitely Suslinian.

Theorem 24 [8, Theorem 9.9]. Let $\mathbf{X}=\left\{X_{a}, p_{a b}, A\right\}$ be a $\sigma$-directed inverse system of rim-finite continua with surjective bonding mappings. Then $X=\lim \mathbf{X}$ is a rim-finite continuum.

Theorem 25. Let $\mathbf{X}=\left\{X_{a}, p_{a b}, A\right\}$ be a $\sigma$-directed inverse system of compact spaces with surjective bonding mappings and limit $X$. Let $Y$ be a metric compact space. For each surjective mapping $f: X \rightarrow Y$ there exists an $a \in A$ such that for each $b \geq a$ there exists a mapping $g_{b}$ : $X_{b} \rightarrow Y$ such that $f=g_{b} p_{b}$.

Proof: Let $\mathcal{B}$ be a countable basis of $Y$ and let $\mathcal{V}$ be a collection of all finite subfamilies of $\mathcal{B}$ which cover $X$. Clearly, $\operatorname{card}(\mathcal{V})=\aleph_{0}$. Hence, $\mathcal{V}=\left\{\mathcal{V}_{n}: n \in \mathbb{N}\right\}$. For each $\mathcal{V}_{n} f^{-1}\left(\mathcal{V}_{n}\right)=\left\{f^{-1}(U): U \in \mathcal{V}_{n}\right\}$ is a covering of $X$. There exists an $a(n) \in A$ such that for each $b \geq a(n)$ there is a cover $\mathcal{V}_{n b}$ of $X_{b}$ with $p_{b}^{-1}\left(\mathcal{V}_{n b}\right) \prec f^{-1}\left(\mathcal{V}_{n}\right)$. From the $\sigma$-directedness of $A$ it follows that there is an $a \in A$ such that $a \geq a(n), n \in \mathbb{N}$. Let $b \geq a$. We claim that $f\left(p_{b}^{-1}\left(x_{b}\right)\right)$ is degenerate. Suppose that there exists a pair $u, v$ of distinct points of $Y$ such that $u, v \in f\left(p_{b}^{-1}\left(x_{b}\right)\right)$. Then there exists a pair $x, y$ of distinct points of $p_{b}^{-1}\left(x_{b}\right)$ such that $f(x)=u$ and $f(y)=v$. Let $U, V$ be a pair of disjoint open sets of $Y$ such that $u \in U$ and $v \in V$. Consider the covering $\{U, V, Y \backslash\{u, v\}\}$. There exists a covering $\mathcal{V}_{n} \in \mathcal{V}$ such that $\mathcal{V}_{n} \prec\{U, V, X \backslash\{u, v\}\}$. We infer that there is a covering $\mathcal{V}_{n b}$ of $X_{b}$ such that $p_{b}^{-1}\left(\mathcal{V}_{n b}\right) \prec f^{-1}\left(\mathcal{V}_{n}\right)$. It follows that $p_{b}(x) \neq p_{b}(y)$ since $x$ and $y$ lie in the disjoint members of the covering $f^{-1}\left(\mathcal{V}_{n}\right)$. This is impossible since $x, y \in p_{b}^{-1}\left(x_{b}\right)$. Thus, $f\left(p_{b}^{-1}\left(x_{b}\right)\right)$ is degenerate. Now we define $g_{b}: X_{b} \rightarrow Y$ by $g_{b}\left(x_{b}\right)=f\left(p_{b}^{-1}\left(x_{b}\right)\right)$. It is 
clear that $g_{b} p_{b}=f$. Let us prove that $g_{b}$ is continuous. Let $U$ be open in $Y$. Then $g_{b}^{-1}(U)$ is open since $p_{b}^{-1}\left(g_{b}^{-1}(U)\right)=f^{-1}(U)$ is open and $p_{b}$ is quotient (as a closed mapping).

Theorem 26. Let $\mathbf{X}=\left\{X_{a}, p_{a b}, A\right\}$ be a $\sigma$-directed inverse system of compact spaces with limit $X$. A closed subspace $Y$ of $X$ is metrizable if and only if there exists an $a \in A$ such that $p_{b} \mid Y: Y \rightarrow p_{b}(Y)$ is a homeomorphism for each $b \geq a$.

Proof: Consider the inverse system $\mathbf{Y}=\left\{p_{a}(Y), p_{a b} \mid p_{b}(Y), A\right\}$ with limit $Y$ and the identity mapping $i: Y \rightarrow Y$. Apply Theorem 25 .

Theorem 27 [3, Corollary 3]. Let $\mathbf{X}=\left\{X_{a}, p_{a b}, A\right\}$ be a $\sigma$-directed inverse system of hereditarily locally connected continua $X_{a}$. Then $X=$ $\lim \mathbf{X}$ is hereditarily locally connected.

The following lemma follows from Theorem 10 and Corollary on p. 69 of [14]. See also [12, Lemma 3.5].

Lemma 28. Let $\mathbf{X}=\left\{X_{a}, p_{a b}, A\right\}$ be an inverse system of compact spaces with monotone bonding surjections, $X=\lim \mathbf{X}, Y$ be a compact space and $m_{a}: Y \rightarrow X_{a}, a \in A$, be monotone surjections such that $m_{a}=$ $p_{a b} m_{b}$ for any $a, b \in A, a \leq b$. Moreover, let $m: Y \rightarrow X$ denote the map induced by $m_{a}, a \in A$. Then $m$ is also a monotone surjection. Moreover, each projection $p_{a}: X \rightarrow X_{a}, a \in A$, is a monotone surjection.

Let $\mathbf{X}=\left\{X_{a}, p_{a b}, A\right\}$ be an inverse system. For each infinite subset $\Delta_{0}$ of $(A, \leq)$ we define sets $\Delta_{n}, n=0,1, \ldots$, by the inductive rule $\Delta_{n+1}=$ $\Delta_{n} \bigcup\left\{m(x, y): x, y \in \Delta_{n}\right\}$, where $m(x, y)$ is a member of $A$ such that $x, y \leq m(x, y)$. Let $\Delta=\bigcup\left\{\Delta_{n}: n \in \mathbb{N}\right\}$. It is clear that $\operatorname{card}(\Delta)=$ $\operatorname{card}\left(\Delta_{0}\right)$. Moreover, $\Delta$ is directed by $\leq[\mathbf{8}$, Lemma 9.2]. For each directed set $(A, \leq)$ we define

$$
A_{\sigma}=\left\{\Delta: \emptyset \neq \Delta \subset A, \operatorname{card}(\Delta) \leq \aleph_{0} \text { and } \Delta \text { is directed by } \leq\right\} .
$$

Then $\mathrm{A}_{\sigma}$ is $\sigma$-directed by inclusion [8, Lemma 9.3]. If $\Delta \in A_{\sigma}$, let $\mathbf{X}^{\Delta}=\left\{X_{b}, p_{b b^{\prime}}, \Delta\right\}$ and $X_{\Delta}=\lim \mathbf{X}^{\Delta}$. If $\Delta, \Gamma \in A_{\sigma}$ and $\Delta \subseteq \Gamma$, let $p_{\Delta \Gamma}: X_{\Gamma} \rightarrow X_{\Delta}$ denote the map induced by the projections $p_{\delta}^{\Gamma}$ : $X_{\Gamma} \rightarrow X_{\delta}, \delta \in \Delta$, of the inverse system $X^{\Gamma}$. Now, we have the following theorem.

Theorem 29 [8, Theorem 9.4]. If $\mathbf{X}=\left\{X_{a}, p_{a b}, A\right\}$ is an inverse system, then $\mathbf{X}_{\sigma}=\left\{X_{\Delta}, p_{\Delta \Gamma}, A_{\sigma}\right\}$ is a $\sigma$-directed inverse system and $\lim \mathbf{X}$ and $\lim \mathbf{X}_{\sigma}$ are canonically homeomorphic. 
Theorem 30. Let $X$ be a compact space. There exists a $\sigma$-directed inverse system $\mathbf{X}=\left\{X_{a}, p_{a b}, A\right\}$ of compact metric spaces $X_{a}$ and surjective bonding mappings $p_{a b}$ such that $X$ is homeomorphic to $\lim \mathbf{X}$.

Proof: See [6, pp. 152, 164].

Theorem 31. If $X$ is a locally connected compact space, then there exists an inverse system $\mathbf{X}=\left\{X_{a}, p_{a b}, A\right\}$ such that each $X_{a}$ is a metric locally connected compact space, each $p_{a b}$ is a monotone surjection and $X$ is homeomorphic to $\lim \mathbf{X}$. Conversely, the inverse limit of such system is always a locally connected compact space.

Proof: See [6, p. 163, Theorem 2].

Theorem 32 [11, Corollary 2.9]. If $X$ is a hereditarily locally connected continuum, then there exists a $\sigma$-directed inverse system $\mathbf{X}=$ $\left\{X_{a}, p_{a b}, A\right\}$ such that each $X_{a}$ is a metrizable hereditarily locally connected continuum, each $p_{a b}$ is a monotone surjection and $X$ is homeomorphic to $\lim \mathbf{X}$.

Let $X$ be a non-degenerate locally connected continuum. A subset $Y$ of $X$ is said to be a cyclic element of $X$ if $Y$ is connected and maximal with respect to the property of containing no separating point of itself. A cyclic element of locally connected continuum is again a locally connected continuum. We let

$$
\mathbf{L}_{X}=\{Y \subset X: Y \text { is a non-degenerate cyclic element of } X\} \text {. }
$$

Lemma 33 [8, Lemma 2.2]. If $C$ is a connected subset of $X$ and $Y \in \mathbf{L}_{X}$, then $C \bigcap Y$ is connected (possibly void).

Lemma 34 [8, Lemma 2.3]. If $f: X \rightarrow X^{\prime}$ is a monotone surjection, then for each $Y^{\prime} \in \mathbf{L}_{X^{\prime}}$ there exists $Y \in \mathbf{L}_{X}$ such that $Y^{\prime} \subseteq f(Y)$. In particular, $\mathbf{L}_{X}$ is non-empty if $\mathbf{L}_{X^{\prime}}$ is non-empty.

Let $Z$ be a cyclic element of $X$. For each component $J$ of $X \backslash Z$, let $\operatorname{Bd}(J)=\left\{z_{J}\right\}$. We define a mapping [8, p. 5] $\rho: X \rightarrow Z$ such that $\rho(x)=x$ if $x \in Z$ and $\rho(x)=z_{J}$ if $x \in J$.

The mapping $\rho$ is a monotone continuous retraction. It is called the canonical retraction of $X$ onto $Z$. 
Theorem 35 [8, Theorem 2.7]. Let $Y$ be a cyclic locally connected continuum and $\mathcal{S}=\left(Y_{\gamma}, f_{\gamma \gamma^{\prime}}, \Gamma\right)$ an inverse system such that $Y=\lim$ inv $\mathcal{S}$ and each bonding mapping $f_{\gamma \gamma^{\prime}}$ is a monotone surjection. For each $\gamma \in$ $\Gamma$, let $Z_{\gamma}$ be either a cyclic element of $Y_{\gamma}$ or a one-point subset of $Y_{\gamma}$. Let $\rho_{\gamma}: Y_{\gamma} \rightarrow Z_{\gamma}$ denote the canonical retraction if $Z_{\gamma}$ is non-degenerate, and otherwise let $\rho_{\gamma}$ be the constant map. Suppose that some $Z_{\gamma_{0}}$ is non-degenerate, and that $Z_{\gamma} \subseteq f_{\gamma \gamma^{\prime}}\left(Z_{\gamma^{\prime}}\right)$ for all $\gamma \leq \gamma^{\prime} \in \Gamma$. Let $g_{\gamma \gamma^{\prime}}=$ $\rho_{\gamma} \circ\left(f_{\gamma \gamma^{\prime}} \mid Z_{\gamma^{\prime}}\right)$ for all $\gamma \leq \gamma^{\prime} \in \Gamma$. Then each $g_{\gamma \gamma^{\prime}}: Z_{\gamma^{\prime}} \rightarrow Z_{\gamma}$ is a monotone surjection and $Y=\operatorname{liminv}\left(Z_{\gamma}, g_{\gamma \gamma^{\prime}}, \Gamma\right)$.

Acknowledgement. The author is grateful to the referee for his help and valuable suggestions.

\section{References}

1. J. L. Cornette, "Image of a Hausdorff arc" is cyclically extensible and reducible, Trans. Amer. Math. Soc. 199 (1974), 253-267.

2. R. Engelking, "General Topology," PWN, Warszawa, 1977.

3. G. R. Gordh, JR. AND S. MARdešIć, Characterizing local connectedness in inverse limits, Pacific J. Math. 58 (1975), 411-417.

4. I. LONČAR, Inverse limit of continuous images of arcs, $Z b$. Rad. (Varaždin) 2(23) (1997), 47-60.

5. T. MaćKowiak, Continuous mappings on continua, Dissertationes Math. (Rozprawy Mat.) 158 (1979), 95 pp.

6. S. MARDEŠIĆ, Locally connected, ordered and chainable continua, Rad Jugoslav. Akad. Znan. Umjet. Odjel Mat. Fiz. Tehn. Nauke 319 (1961), 147-166.

7. F. MAEDA, "Kontinuierliche geometrien," Springer-Verlag, Berlin, 1958.

8. J. Nikiel, H. M. Tunchli And E. D. Tymchatyn, Continuous images of arcs and inverse limit methods, Mem. Amer. Math. Soc. 104 (1993), 80 pp.

9. J. Nikiel, L. B. Treybig And H. M. TuncAli, Local connectivity and maps onto non-metrizable arcs, Internat. J. Math. Math. Sci. 20 (1997), 681-688.

10. J. Nikiel, Continuous images of arcs, Proc. Amer. Math. Soc. 103 (1988), 961-968.

11. J. NikiEL, The Hahn-Mazurkiewicz theorem for hereditarily locally connected continua, Topology Appl. 32 (1989), 307-323. 
12. J. Nikiel, A general theorem on inverse systems and their limits, Bull. Polish Acad. Sci. Math. 37 (1989), 127-136.

13. J. NikiEL, Locally connected curve viewed as inverse limits, Fund. Math. 133 (1989), 125-134.

14. E. PuZIO, Limit mappings and projections of inverse systems, Fund. Math. 80 (1973), 57-73.

15. J. N. Simone, Continuous images of ordered compacta and hereditarily locally connected continua, Colloq. Math. 40 (1978), 77-84.

16. J. N. Simone, Concerning hereditarily locally connected continua, Colloq. Math. 39 (1978), 243-251.

17. J. N. Simone, Suslinian images of ordered compacta and a totally nonmetric Hahn-Mazurkiewicz theorem, Glas. Mat. Ser. III 13(33) (1978), 343-346.

18. L. B. Treybig, Concerning continua which are continuous image of compact ordered spaces, Duke Math. J. 32 (1965), 417-422.

19. L. B. Treybig, Separation by finite sets in connected, continuous images of ordered compacta, Proc. Amer. Math. Soc. 74 (1979), 326-328.

20. G. T. Whyburn, "Analytic Topology," Amer. Math. Soc., Providence, R.I., 1963.

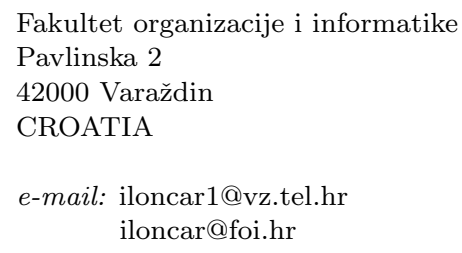

Primera versió rebuda el 8 de juny de 1998, darrera versió rebuda el 18 de maig de 1999 